\title{
ANHARMONIC POLYNOMIAL GENERALIZATIONS OF THE NUMBERS OF BERNOULLI AND EULER*
}

BY

E. T. BELL

We consider twelve infinite systems of polynomials in $z$ which for $z=1$ degenerate either to the numbers of Bernoulli or Euler, or to others simply dependent upon these. The first part proceeds from the definition of anharmonic polynomials to the specific twelve systems discussed; the second presents an adaptation of the symbolic calculus of Blissard and Lucas in sufficient detail for rapidly developing a simple isomorphism between the algebra of the polynomials and that of the twelve elliptic functions sn, cn, ns, nc, sc, $\cdots$ of Glaisher, and the third contains a short selection from the simpler algebraic and congruential relations between the polynomials. Incidentally there is pointed out in the second part a new interpretation of Kronecker's work on certain symmetric functions and their connections with Bernoulli's numbers. Owing to the length of the paper the development stops short of the quadratic transformation of the polynomials which corresponds to the transformation of the second order in elliptic functions, but the material given is a necessary foundation for all higher transformations. For the same reason only prime moduli are considered in the congruences, although the case in which the modulus is a power of a prime can be treated in essentially the same way, but at greater length. All references are at the end of the paper.

\section{ANHARMonic POLYNomials}

1. With each of the substitutions $\sigma^{\prime}$ of the cross ratio group on $z$,

$$
\begin{array}{rlrl}
1 & =(z, z), & \alpha^{\prime}=(z, 1 /(1-z)), & \beta^{\prime}=(z,(z-1) / z), \\
\gamma^{\prime}=(z, 1 / z), & \delta^{\prime}=(z, 1-z), & \epsilon^{\prime}=(z, z /(z-1)),
\end{array}
$$

associate a multiplier $\sigma^{\prime \prime}$ as follows,

$$
\begin{array}{clrl}
1, & \alpha^{\prime \prime}=(z-1)^{n}, & \beta^{\prime \prime}=(-z)^{n}, \\
\gamma^{\prime \prime}=z^{n}, & \delta^{\prime \prime}=(-1)^{n}, & \epsilon^{\prime \prime}=(1-z)^{n},
\end{array}
$$

$n$ being an integer $\geqq 0$. From these define six linear substitutions upon the coefficients $a_{0}, a_{1}, \cdots, a_{n}$, with $a_{n} \neq 0$, of the polynomial

$$
A(z)=a_{0}+a_{1} z+a_{2} z^{2}+\cdots+a_{n} z^{n}
$$

* Presented to the Society, San Francisco Section, April 9, 1921. 
by means of the identities

$$
\sigma A(z) \equiv \sigma^{\prime \prime} A\left(\sigma^{\prime} z\right) \equiv a_{\sigma 0}+a_{\sigma 1} z+a_{\sigma 2} z^{2}+\cdots+a_{\sigma n} z^{n},
$$

in which $A\left(\sigma^{\prime} z\right)$ denotes the result of applying $\sigma^{\prime}$ to $A(z)$. Hence $\sigma A(z)$ is the polynomial derived from $A(z)$ by first operating with $\sigma^{\prime}$ and then multiplying the result by $\sigma^{\prime \prime}$. If $a_{0}=0$ the degree of $\sigma A(z)$ is $<n$ for some $\sigma$. The six $\sigma$ 's thus obtained are $1, \alpha, \beta, \gamma, \delta, \epsilon$, where $\alpha$ corresponds to $\alpha^{\prime}, \alpha^{\prime \prime}$, etc.

2. A set of generating relations for the cross ratio group is

$$
\alpha^{\prime 3}=\gamma^{\prime 2}=\left(\alpha^{\prime} \gamma^{\prime}\right)^{2}=1 \text {. }
$$

We have $\alpha A(z)=(z-1)^{n} A\left(\alpha^{\prime} z\right)$; whence

$$
\begin{aligned}
& \alpha^{2} A(z) \equiv \alpha(\alpha A(z))=(z-1)^{n}\left[(z /(1-z))^{n} A\left({\alpha^{\prime}}^{2} z\right)\right], \\
& \alpha^{3} A(z) \equiv \alpha\left(\alpha^{2} A(z)\right)=(z-1)^{n}\left[(1 /(z-1))^{n} A\left({\alpha^{\prime}}^{3} z\right)\right]=A(z) .
\end{aligned}
$$

Hence $\alpha^{3}=1$, and similarly $\gamma^{2}=1,(\alpha \gamma)^{2}=1$, so that the $\sigma$ form a group $\Gamma$ simply isomorphic to the cross ratio group. For convenience of reference we reproduce its multiplication table, which is to be read in the usual way; thus $\alpha \gamma=\delta, \gamma \epsilon=\alpha$, and so on:

\begin{tabular}{l|llllll}
\multicolumn{1}{c}{} & \multicolumn{1}{c}{1} & $\alpha$ & $\beta$ & $\gamma$ & $\delta$ & $\epsilon$ \\
\hline$\alpha$ & $\alpha$ & $\beta$ & 1 & $\delta$ & $\epsilon$ & $\gamma$ \\
$\beta$ & $\beta$ & 1 & $\alpha$ & $\epsilon$ & $\gamma$ & $\delta$ \\
$\gamma$ & $\gamma$ & $\epsilon$ & $\delta$ & 1 & $\beta$ & $\alpha$ \\
$\delta$ & $\delta$ & $\gamma$ & $\epsilon$ & $\alpha$ & 1 & $\beta$ \\
$\epsilon$ & $\epsilon$ & $\delta$ & $\gamma$ & $\beta$ & $\alpha$ & 1
\end{tabular}

3. Obviously $a_{\gamma r}=a_{1 n-r} \equiv a_{n-r}$. Let $\rho, \sigma, \tau$ be substitutions of $\Gamma$ between which there is the relation $\rho \sigma=\tau$, and write $a_{\rho \sigma r}$ for the function of the coefficients $a_{r}$ which is derived from the $a_{\sigma r}$ in the same way that $a_{\rho r}$ is from the $a_{r}$. Then $a_{\rho \sigma r}=a_{r r}$, and the coefficients of the six polynomials are related as shown by the table in $\S 2$. If $\gamma \sigma=\tau$, then $a_{\tau r}=a_{\gamma \sigma r}=a_{\sigma n-r}$. Hence, writing as usual $0 !=1,\left(\begin{array}{l}r \\ 0\end{array}\right)=\left(\begin{array}{l}0 \\ 0\end{array}\right)=1,\left(\begin{array}{l}r \\ s\end{array}\right)=r ! /(s !(r-s) !)$, we have the following forms of the $a_{\sigma r}, r=0,1, s, \cdots, n$, which explicitly define the substitutions of $\Gamma$ :

$$
\begin{aligned}
& a_{1 r}=a_{\gamma n-r}=a_{r}, \\
& a_{\alpha r}=a_{\epsilon n-r}=(-1)^{n-r} \sum_{s=0}^{n-r}\left(\begin{array}{c}
n-s \\
r
\end{array}\right) a_{s}, \\
& a_{\beta r}=a_{\delta n-r}=(-1)^{r} \sum_{s=0}^{r}\left(\begin{array}{c}
n-s \\
r-8
\end{array}\right) a_{n-s},
\end{aligned}
$$

the $a_{\sigma r}$ being given directly by the $\sigma A(z)$ for $\sigma=1, \alpha, \beta$. 
4. The set $A(z), \alpha A(z), \beta A(z), \gamma A(z), \delta A(z), \epsilon A(z)$ is called anharmonic of degree $n, n$ being the highest degree of any polynomial in the set. Any symmetric function of the polynomials $A(z), \sigma A(z)$ is an invariant of the subgroup $1, \sigma(\sigma=\gamma, \delta, \epsilon)$; any symmetric function of $A(z), \alpha A(z)$, $\beta A(z)$ is an invariant of the subgroup $1, \alpha, \beta$; and any symmetric function of $\sigma A(z)(\sigma=1, \alpha, \beta, \gamma, \delta, \epsilon)$ is an invariant of $\Gamma$.

5. When $A(z)$ is reciprocal, $\gamma A(z)=A(z)$. Hence from the multiplication table $\delta A(z)=\alpha A(z), \epsilon A(z)=\beta A(z)$, and in this case the anharmonic set reduces to $A(z), \alpha A(z), \beta A(z)$. We shall call such a set cyclic, and henceforth reserve the term anharmonic for sets that are not cyclic or, what is equivalent, contain no reciprocal polynomial. Any symmetric function of all the members of a cyclic set is invariant for $1, \alpha, \beta$.

6. The $\sigma$ are determined by the coefficients $a_{r}$ as in $\S 3$ and are functions of the degree $n$ of the set. When necessary to designate the polynomial fixing the $\sigma$ we shall speak of the associated $\sigma$ as the cross ratio substitution for that polynomial. To indicate that $A(z)$ belongs to a set of degree $n$ we write $A_{(n)}(z)$, enclosing the $n$ in ( ) to distinguish it from the rank defined in $\S 7$. When it is a question of relations between the coefficients of polynomials of different degrees $(\$ 15)$ the $a_{r}$ may be given double suffixes. Thus

$$
A(z) \equiv A_{(n)}(z) \equiv \sum_{r=0}^{n} a_{r} z^{r} \equiv \sum_{r=0}^{n} a_{n r} z^{r}
$$

are merely different notations for one polynomial, and likewise for

$$
\sigma A(z) \equiv \sigma A_{(n)}(z) \equiv \sum_{r=0}^{n} a_{\sigma r} z^{r} \equiv \sum_{r=0}^{n} a_{\sigma n r} z^{r}
$$

Where there can be no confusion we shall use the simpler forms.

7. Let $x, z$ be independent variables, and put $\sqrt{z}=k, \sqrt{1-z}=k^{\prime}$, $i=\sqrt{-1}$. Let $t, v$ be functions of $x, k$ (or of $x, z$ ) such that

$$
\begin{aligned}
& t(x,-k)=t(x, k)=-t(-x, k), \\
& v(x,-k)=v(x, k)=v(-x, k),
\end{aligned}
$$

and assume (cf. $\S 12$ ) that these functions can be expanded in absolutely convergent power series in $x$ of the form

$$
t(x, k)=\sum_{n=0}^{\infty} \frac{(-1)^{n} x^{2 n+1}}{(2 n+1) !} T_{(n)}(z), \quad v(x, k)=\sum_{n=0}^{\infty} \frac{(-1)^{n} x^{2 n}}{(2 n) !} V_{(n)}(z) .
$$

We now define $T_{(n)}(z)$ arising from an odd function generator $t(x, k)$ to be an odd polynomial of rank $2 n+1$, and $V_{(n)}(z)$ generated by an even function $v(x, k)$ to be an even polynomial of rank $2 n$, and write

$$
T_{(n)}(z) \equiv T_{2 n+1}(z), \quad V_{(n)}(z) \equiv V_{2 n}(z),
$$


so that the degree of a set containing a given polynomial is the greatest integer in half the rank. This change in notation is essential for the further development, as without it the application of the symbolic calculus of Blissard and Lucas is impracticable. Exhibiting the ranks rather than the degrees we have

$$
\begin{aligned}
& t(x, k)=\sum_{n=0}^{\infty} \frac{(-1)^{n} x^{2 n+1}}{(2 n+1) !} T_{2 n+1}(z) \equiv \sin T(z) x, \\
& v(x, k)=\sum_{n=0}^{\infty} \frac{(-1)^{n} x^{2 n}}{(2 n) !} V_{2 n}(z) \equiv \cos V(z) x,
\end{aligned}
$$

the trigonometric forms being the purely symbolic equivalents of the series.

The principles of the symbolic method, which we shall use freely in the sequel, having been fully treated by Blissard, Lucas and Glaisher, in the works cited, we need not recall them here, except to emphasize the caution that in all operations with symbolic powers zero exponents must be included. Thus the first term of $\cos V(z) x$ is $V_{0}(z)$, not unity; the symbolic binomial $(p+q)^{2}=p^{2} q^{0}+2 p^{1} q^{1}+p^{0} q^{2} \equiv p_{2} q_{0}+2 p_{1} q_{1}+p_{0} q_{2}$, not $p_{2}+2 p_{1} q_{1}+q_{2}$; and $(p-p)^{2}=2\left(p_{2} p_{0}-p_{1}^{2}\right)$, obtained from $(p-q)^{2}$ by putting $q=p$ in the final form of the latter.

8. That $\sigma$ is one of the cross ratio substitutions for $A_{n}(z)$ may be indicated by writing $\sigma_{n}$, but for simplicity we shall put $\sigma_{n} A_{n}(z) \equiv \sigma A_{n}(z)$. Analogously to the $\mathrm{sn}, \mathrm{cn}, \mathrm{ns}, \cdots$ notation for elliptic functions we denote each of the six polynomials $1 A_{n}(z) \equiv A_{n}(z), \alpha A_{n}(z), \beta A_{n}(z), \cdots, \epsilon A_{n}(z)$ by a double letter symbol $1 A \equiv A, \alpha A, \beta A, \cdots, \epsilon A$, and in any such $\sigma A$ regard the $\sigma, A$ as inseparable. In Blissard's method $A_{n}(z)$ is written $A^{n}(z)$, or $A^{n}$ when $z$ is understood, the exponent being purely symbolic, and $A$ is called an umbra. Similarly we now have $(\sigma A)^{n} \equiv \sigma A_{n}$, since $\sigma A$ is one symbol, and our umbræ are double-letter symbols $\sigma A$. It is important to note once for all that $(\sigma A)^{n}$ is not $\sigma^{n} A_{n}$ in which $\sigma^{n}$ has the usual meaning as a power of a substitution.

9. We require the operations transforming the generators of $T, V$ into those of $\sigma T, \sigma V$ respectively. Let $f(x, k)$ denote an arbitrary function of $x, k$, and $\Omega_{i}$ an operator which applied to $f$ transforms it as follows:

$$
\Omega_{i} f(x, k)=\phi_{i}(k) f\left(x \psi_{i}(k), \chi_{i}(k)\right) .
$$

We regard $\Omega_{i}$ as a tripartite operator,

$$
\Omega_{i} \equiv\left\{\phi_{i}(k), \psi_{i}(k), \chi_{i}(k)\right\},
$$

which replaces $k$ in $f(x, k)$ by $\chi_{i}(k), x$ by $x \psi_{i}(k)$, and multiplies the function thus transformed by $\phi_{i}(k)$. Hence the product $\Omega_{j} \Omega_{i}$ in which $\Omega_{i}$ is applied first is

$$
\left\{\phi_{j}(k) \phi_{i}\left(\chi_{j}(k)\right), \quad \psi_{j}(k) \psi_{i}\left(\chi_{j}(k)\right), \quad \chi_{i}\left(\chi_{j}(k)\right)\right\}
$$


and if $f(x, k)$ is odd in $x$, even in $k$,

$$
\{\phi, \psi, \chi\}=\{-\phi,-\psi, \chi\}=\{\phi, \psi,-\chi\} ;
$$

while if $f(x, k)$ is even in both $x$ and $k$,

$$
\{\phi, \psi, \chi\}=\{\phi,-\psi, \chi\}=\{\phi, \psi,-\chi\} .
$$

Let $\sigma^{\prime}, \sigma$ denote any cross ratio substitutions for $T_{2 n+1}(z), V_{2 n}(z)$ respectively, so that symbolically

$$
\begin{aligned}
& \sin \sigma^{\prime} T(z) x \equiv \sum_{n=0}^{\infty} \frac{(-1)^{n} x^{2 n+1}}{(2 n+1) !} \sigma^{\prime} T_{2 n+1}(z), \\
& \cos \sigma V(z) x \equiv \sum_{n=0}^{\infty} \frac{(-1)^{n} x^{2 n}}{(2 n) !} \sigma V_{2 n}(z)
\end{aligned}
$$

and designate by $s^{\prime}, s$ operators such that

$$
s^{\prime} t(x, k)=\sin \sigma^{\prime} T(z) x, \quad s v(x, k)=\cos \sigma V(z) x .
$$

Then by inspection of the series, the Greek and Latin letters $(\alpha, a)$, $\left(\alpha^{\prime}, a^{\prime}\right), \cdots,(\sigma, s),\left(\sigma^{\prime}, s^{\prime}\right)$ corresponding, we have

$$
\begin{aligned}
1 & =\{1,1, k\}, & 1 & =\{1,1, k\}, \\
a^{\prime} & =\left\{-i / k^{\prime}, i k^{\prime}, 1 / k^{\prime}\right\}, & & a=\left\{1, i k^{\prime}, 1 / k^{\prime}\right\}, \\
b^{\prime} & =\left\{-i / k, i k, i k^{\prime} / k\right\}, & b & =\left\{1, i k, i k^{\prime} / k\right\}, \\
c^{\prime} & =\{1 / k, k, 1 / k\}, & c & =\{1, k, 1 / k\}, \\
d^{\prime} & =\left\{-i, i, k^{\prime}\right\}, & d & =\left\{1, i, k^{\prime}\right\}, \\
e^{\prime} & =\left\{1 / k^{\prime}, k^{\prime}, i k / k^{\prime}\right\} ; & e & =\left\{1, k^{\prime}, i k / k^{\prime}\right\} .
\end{aligned}
$$

From the definitions the $s^{\prime}$ form a group $G^{\prime}$ which is simply isomorphic to $\Gamma$ and whose multiplication table is obtained from that in $\$ 2$ upon replacing each Greek letter by its accented Latin correspondent; the multiplication table for the group $G$ of operators $s$ is obtained from $G^{\prime}$ by suppressing accents. The explicit forms of the operators given above can be verified easily on combining them according to the formulas developed for the $\Omega_{i}$, noting that the $s^{\prime}$ refer to an odd, and the $s$ to an even function.

10. Thus far $s^{\prime}$ has been applied only to the odd $t(x, k)$, and $s$ to the even $v(x, k)$. The following cases are of equal importance, and they may be seen at once from the definitions. If

$$
\begin{array}{ll}
s^{\prime} \equiv\left\{\phi^{\prime}(k), \psi^{\prime}(k), \chi^{\prime}(k)\right\}, & \text { then } \quad s=\left\{1, \psi^{\prime}(k), \chi^{\prime}(k)\right\} ; \\
s^{\prime} v(x, k)=\phi^{\prime}(k) \cdot s v(x, k), & s t(x, k)=\frac{1}{\phi^{\prime}(k)} \cdot s^{\prime} t(x, k) .
\end{array}
$$


11. We now specialize the even $V$ in each of two ways and the odd $T$ in one way, getting in all for each integral degree $n \geqq 0$ a system of twelve polynomials distributed into two cyclic sets and one anharmonic. Indicating that the modulus of the elliptic functions is $k$ by writing $\operatorname{sn}(x, k)$, etc., we define the fundamental polynomials $S, C, P$ and their anharmonic transforms $\tau^{\prime} S, \sigma C, \sigma P$ by their symbolic generators,

$$
\begin{aligned}
\operatorname{sn}(x, k) & \equiv \sin S(z) x, & s^{\prime} \operatorname{sn}(x, k) & \equiv \sin \sigma^{\prime} S(z) x, \\
\mathrm{cn}(x, k) & \equiv \cos C(z) x, & s \mathrm{cn}(x, k) & \equiv \cos \sigma C(z) x, \\
x \mathrm{~ns}(x, k) & \equiv \cos P(z) x ; & s x \mathrm{~ns}(x, k) & \equiv \cos \sigma P(z) x .
\end{aligned}
$$

From the elements of elliptic functions the coefficients in $S_{2 n+1}(z), C_{2 n}(z)$ are positive integers, $C_{2 n}(z)$ is of degree $n-1$ in $z$, and $S_{2 n+1}$ is a reciprocal polynomial of degree $n$ in $z$. Hence $P_{2 n}(z)$ is a reciprocal polynomial, since $x$ ns $x=x / \operatorname{sn} x$. Therefore, omitting ranks from the notation, the coefficients in each of the sets $\sigma^{\prime} S(z), \sigma C(z)$ are integers, those in $\sigma P(z)$ are rational but not integral, and of the three sets $\sigma^{\prime} S(z), \sigma P(z)$ are cyclic, $\sigma C(z)$ is anharmonic.

12. If for all values of $z$ whose absolute value does not exceed a constant different from zero the algebraic relation $F(z)=0$ holds, then it is easily seen that $F\left(z^{\prime}\right)=0$ is an identity in arbitrary $z^{\prime}$. Hence in all polynomial formulas we consider $z$ arbitrary, it being understood that when $F(z)$ is regarded as a coefficient in an infinite series $z$ is such as to render the series absolutely convergent (all the series discussed have radius of convergence $>0$ ), but that in all other connections $z$ is arbitrary.

13. For convenience of comparison with Glaisher's grouping of the elliptic functions into triads we give the complete set of generators:

$$
\begin{array}{ll}
\text { sn }(x, k)=\sin S(z) x, & x \text { ns }(x, k)=\cos P(z) x, \\
\text { cn }(x, k)=\cos C(z) x, & x \operatorname{cs}(x, k)=\cos \alpha P(z) x, \\
\text { dn }(x, k)=\cos \gamma C(z) x ; & x \text { ds }(x, k)=\cos \beta P(z) x ; \\
\text { dc }(x, k)=\cos \alpha C(z) x, & \operatorname{cd}(x, k)=\cos \epsilon C(z) x, \\
\text { nc }(x, k)=\cos \delta C(z) x, & \text { sd }(x, k)=\sin \beta S(z) x, \\
\text { sc }(x, k)=\sin \alpha S(z) x ; & \text { nd }(x, k)=\cos \beta C(z) x .
\end{array}
$$

Glaisher points out that of the four triads the second is the most symmetrical, and that it should be taken as a basis for the jacobian elliptic functions rather than the traditional first. From the present point of view the second triad frequently appears to be wholly anomalous: while the sets $\sigma^{\prime} S(z), \sigma C(z)$ in many significant ways can be regarded as forming one complete system, the 
set $\sigma P(z)$ stands apart in the majority of relations of a specific type. We see in a moment that $\sigma P(z)$ is related to the Bernoulli numbers, $\sigma^{\prime} S(z), \sigma C(z)$ to those of Genocchi and Euler respectively. Hence we have another instance of that superficial similarity and radical difference between the numbers of Bernoulli and Euler which has often been remarked.

14. When $z=1$ and hence $k=1$ the elliptic functions degenerate to circular functions of the gudermannian of $x$. From the values of these as given by Cayley, p. 59, and the symbolic generators for the $B, E, G, R$ (numbers of Bernoulli, Euler, Genocchi and Lucas) in Lucas 5, p. 262, we find at once

$$
\begin{aligned}
x \text { ns }(x, 1) & =i x \cot i x=\cos 2 B i x, \\
\text { cn }(x, 1) & =\sec i x=\cos \text { Eix, } \\
\operatorname{dn}(x, 1) & =\sec i x=\cos E i x, \\
2 x \operatorname{sn}(x, 1) & =-2 i x \tan i x=-\cos 2 G i x, \\
x & \text { ds }(x, 1)=i x \operatorname{cosec} i x=2 \cos \text { Rix, } \\
x \operatorname{cs}(x, 1) & =i x \operatorname{cosec} i x=2 \cos R i x .
\end{aligned}
$$

The even suffix notation of Lucas is used for all $B, E, G, R$, and the last two are defined by

$$
G_{2 n}=2\left(1-2^{2 n}\right) B_{2 n}, \quad R_{2 n}=\left(1-2^{2 n-1}\right) B_{2 n} .
$$

We require a fifth system of numbers $H_{2 n+1}$, the so-called tangent coefficients, shown presently to be integers $>0$,

$$
H_{2 n+1}=\frac{(-1)^{n+1}}{n+1} 2^{2 n} G_{2 n+2}=\frac{(-1)^{n}}{n+1} 2^{2 n+1}\left(2^{2 n+2}-1\right) B_{2 n+2} .
$$

Comparing coefficients of like powers of $x$ in the generators above with those in $\S 13$ we have for $n \geqq 0, r>0$ (note that in each case the degenerate form is expressed as a function of the rank $2 n$ or $2 n+1)$,

$$
\begin{aligned}
S_{2 n+1}(1) & =H_{2 n+1}, & P_{2 n}(1) & =(-1)^{n} 2^{2 n} B_{2 n}=(2 i)^{2 n} B_{2 n}, \\
\alpha S_{2 n+1}(1) & =(-1)^{n}=-i \cdot i^{2 n+1}, & \alpha P_{2 n}(1) & =2(-1)^{n} R_{2 n}=2 i^{2 n} R_{2 n}, \\
\beta S_{2 n+1}(1) & =(-1)^{n}=-i \cdot i^{2 n+1} ; & \beta P_{2 n}(1) & =2(-1)^{n} R_{2 n}=2 i^{2 n} R_{2 n} \\
C_{2 n}(1) & =(-1)^{n} E_{2 n}=i^{2 n} E_{2 n}, & \gamma C_{2 n}(1) & =(-1)^{n} E_{2 n}=i^{2 n} E_{2 n}, \\
\alpha C_{0}(1) & =1, \alpha C_{2 r}(1)=0, & \delta C_{2 n}(1) & =(-1)^{n}=i^{2 n}, \\
\beta C_{2 n}(1) & =(-1)^{n}=i^{2 n} ; & \epsilon C_{0}(1) & =1, \quad \epsilon C_{2 r}(1)=0
\end{aligned}
$$

The coefficients in $S_{2 n+1}(z), C_{2 n}(z)$ being integers $>0$, so also are $H_{2 n+1}$, $(-1)^{n} E_{2 n}$. 
15. An identity between some or all of the twelve elliptic functions implies and is implied by the identity between polynomials which is obtained upon equating coefficients of like powers of the argument $x$, and as degenerate cases for $z=1$ of these identities we have relations between the numbers $B, E, H, R$. The elliptic identities may conveniently be segregated into classes according to the groups $G, G^{\prime}$ of $\S 9$. All the identities arising from a given one by successive applications of the operations of $G$ belong to the first class, all those similarly derived by means of $G^{\prime}$ belong to the second, and there are subsidiary classes corresponding to the cyclic subgroups of orders 3,2 . Polynomial identities derived from elliptic identities of a given class belong to one class. Elliptic identities are further subdivided into types according to the degree of the identity in $\mathrm{sn}, \mathrm{cn}, \cdots$, and the derived polynomial relations are similarly subdivided, the degenerate cases being included. By means of the table in $\S 2$ and those next given, together with the formulas of $\S 10$, we write down immediately from any elliptic identity all others of the same type, and hence on replacing the elliptic functions by their symbolic trigonometric equivalents from $\S 13$ we at once infer all the polynomial relations of one type. It is clear that once the elliptic identity is given the rest of the process demands very little labor. In the next part we develop the symbolic method proper to the subject, and this still further reduces the algebra.

Consider any one of the polynomial relations. This is an identity in $z$ and hence, $z$ being arbitrary, it is equivalent to a set of identical relations between the coefficients of the several polynomials. The coefficients of $S_{2 n+1}(z)$ are $n+1$ integers $>0$ into which $H_{2 n+1}$ is partitioned; those of $C_{2 n}(z)$ are $n$ integers $>0$ into which $(-1)^{n} E_{2 n}$ is partitioned, and those of $P_{2 n}(z)$ are a rational but not integral partition of $(-1)^{n} 2^{2 n} B_{2 n}$. From the explicit values of the transformed coefficients in $\S 3$ the coefficients of the transforms of the fundamental polynomials $S, C, P$ are known in terms of the foregoing partitions, and hence the polynomial relation is equivalent to a theorem concerning partitions of a certain kind of the numbers $B, E, H$ of various ranks. Similar remarks apply to congruences between such of the polynomials as take integral values when $z$ is an integer. This aspect is not further elaborated here, as its complete discussion presupposes a knowledge of the arithmetical form of the coefficients in $C_{2 n}(z), S_{2 n+1}(z) .^{*}$ It is not difficult to give implicit arithmetical definitions of these coefficients, but this is not what is required.

* The well known method of Hermite $(10$, pp. 265,269$)$ for calculating $C_{2 n}(z), S_{2 n+1}(z)$ does not give the required information, as recognized by Hermite himself $(12$, p. 237). His second solution (ibid.), as pointed out by the editors of his works, unfortunately is erroneous, and even if it were correct it is difficult to see what the general coefficient would be from the forms of those given. Writers on elliptic functions seem to have overlooked Hermite's remarks in the second citation, and to have assumed that his first paper is sufficient. 
16. From the multiplication tables of $G, G^{\prime}$ combined with the results of the linear transformations of $\mathrm{sn}(x, k), \mathrm{cn}(x, k)$ (as given for example by Glaisher $\left(6\right.$, p. 120)) we have the following for the generators of the $\sigma^{\prime} S, \sigma P$ :

\begin{tabular}{|c|c|c|c|c|c|c|}
\hline & sn & sc $\quad$ sd & & $x$ ns $x$ & $x \operatorname{cs} x$ & $x \mathrm{ds} x$ \\
\hline 1 & sn & sc $\quad$ sd & 1 & $x$ ns $x$ & $x \operatorname{cs} x$ & $x \mathrm{ds} x$ \\
\hline$a^{\prime}$ & $\mathrm{sc}$ & sd sn & $a^{\prime}$ & $x \operatorname{cs} x$ & $x \mathrm{ds} x$ & $x$ ns $x$ \\
\hline$b^{\prime}$ & sd & sn $\quad s c$ & $b^{\prime}$ & $x \mathrm{ds} x$ & $x$ ns $x$ & $x \operatorname{cs} x$ \\
\hline
\end{tabular}

the argument being $x$ and the modulus $k$. Since each set is cyclic the transformations $c^{\prime}, d^{\prime}, e^{\prime}$ are respectively identical with $1, a^{\prime}, b^{\prime}$. Corresponding to $\S 5$ we have: any symmetric function of all the members of either of these cyclic sets is an invariant of $1, a^{\prime}, b^{\prime}$.

For the anharmonic set the table is

\begin{tabular}{l|llllll}
\multicolumn{1}{c}{} & cn & dc & nd & dn & nc & cd \\
1 & cn & dc & nd & dn & nc & cd \\
$a$ & dc & nd & cn & nc & cd & dn \\
$b$ & nd & cn & dc & cd & dn & nc \\
$c$ & dn & cd & ne & cn & nd & dc \\
$d$ & nc & dn & cd & dc & cn & nd \\
$e$ & cd & nc & dn & nd & dc & cn
\end{tabular}

The modulus and argument in each case are $k, x$. Any symmetric function of the members of the following pairs $(c n, d n),(d c, d n),(d n, n d)$ is an invariant of the group $(1, c),(1, d)$ or $(1, e)$ respectively; any symmetric function of $\mathrm{dn}, \mathrm{nc}, \mathrm{cd}$ is an invariant of $(1, a, b)$, and any symmetric function of $\mathrm{cn}, \mathrm{dc}, \mathrm{nd}, \mathrm{dn}, \mathrm{nc}, \mathrm{cd}$ is an invariant of $G$.

17. One example will suffice to show how all the relations of a given type may be written down from one of them. When $k$ or $k^{\prime}$ occurs as a factor it is replaced in the final result by its $z$-equivalent. The modulus being $k$, consider $\operatorname{sn}^{2} x+\operatorname{cn}^{2} x=1$. From this, since $\phi(k)=1$ for each $s$,

$$
(s \operatorname{sn} x)^{2}+(s \operatorname{cn} x)^{2}=1 .
$$

Putting $s=a$ we have, by $\S 10$ and the form of $a^{\prime}$ in $\S 9$,

$$
\left(i k^{\prime} a^{\prime} \operatorname{sn} x\right)^{2}+(a \text { cn } x)^{2}=1 ;
$$

and hence from the tables in $\$ 16,-{k^{\prime}}^{2} \mathrm{sc}^{2} x+\mathrm{dc}^{2} x=1$. To illustrate useful processes we shall also consider in detail the effects of operating with $s^{\prime}$.

Let $s^{\prime} \equiv\left\{\phi^{\prime}, \psi^{\prime}, \chi^{\prime}\right\}$. Then

$$
\begin{gathered}
s^{\prime}\left[\operatorname{sn}^{2} x+\operatorname{cn}^{2} x\right]=s^{\prime} 1=\phi^{\prime}(k) ; \\
\phi^{\prime}(k)\left[\operatorname{sn}^{2}\left(x \psi^{\prime}(k), \chi^{\prime}(k)\right)+\operatorname{cn}^{2}\left(x \psi^{\prime}(k), \chi^{\prime}(k)\right)\right]=\phi^{\prime}(k) ;
\end{gathered}
$$




$$
\begin{gathered}
\operatorname{sn}^{2}\left(x \psi^{\prime}(k), \chi^{\prime}(k)\right)+\operatorname{cn}^{2}\left(x \psi^{\prime}(k), \chi^{\prime}(k)\right)=1 ; \\
\frac{1}{{\phi^{\prime 2}}^{2}(k)}\left(s^{\prime} \operatorname{sn}(x, k)\right)^{2}+(s \mathrm{cn}(x, k))^{2}=1,
\end{gathered}
$$

and hence for $s^{\prime}=a^{\prime},-k^{\prime 2} \operatorname{sc}^{2} x+\mathrm{dc}^{2} x=1$.

With a little practice the transform can be written down by inspection in any case directly from $\$ 9,10,16$. Thus the complete set here is

$$
\begin{aligned}
\operatorname{cn}^{2} x+\operatorname{sn}^{2} x & =1, \\
\mathrm{dc}^{2} x-k^{\prime 2} \operatorname{sc}^{2} x & =1, \\
\operatorname{nd}^{2} x-k^{2} \operatorname{sd}^{2} x & =1, \\
\mathrm{dn}^{2} x+k^{2} \operatorname{sn}^{2} x & =1, \\
\operatorname{nc}^{2} x-\operatorname{sc}^{2} x & =1, \\
\operatorname{cd}^{2} x+k^{\prime 2} \operatorname{sd}^{2} x & =1 ;
\end{aligned}
$$$$
\left(b \text { or } b^{\prime}\right) \text {, }
$$$$
\left(c \text { or } c^{\prime}\right) \text {, }
$$$$
\left(d \text { or } d^{\prime}\right) \text {, }
$$$$
\left(e \text { or } e^{\prime}\right) \text {, }
$$

whence the final forms

$$
\begin{aligned}
\cos ^{2} C(z) x+\sin ^{2} S(z) x & =1, \\
\cos ^{2} \alpha C(z) x-(1-z) \sin ^{2} \alpha S(z) x & =1, \\
\cos ^{2} \beta C(z) x-z \sin ^{2} \beta S(z) x & =1, \\
\cos ^{2} \gamma C(z) x+z \sin ^{2} S(z) x & =1, \\
\cos ^{2} \delta C(z) x-\sin ^{2} \alpha S(z) x & =1, \\
\cos ^{2} \epsilon C(z) x+(1-z) \sin ^{2} \beta S(z) x & =1 .
\end{aligned}
$$

Simple rules may be devised for $1 .$. iting down the appropriate multipliers such as $z, 1-z$ above, from the forms of the $s^{\prime} ; s$ and $\S 10$, for the several terms of any symbolic identity when the argument $z$ of the polynomials is transformed by the substitutions of $\Gamma$. As these present no difficulty we omit them.

\section{ISOMORPHIGM WITH ELLIPTIC FUNCTIONS}

18. To find the polynomial relation equivalent to a given elliptic identity we evidently must consider the properties of products of $t$ symbolic sines and $r$ symbolic cosines in the cases $r, t>0 ; r>0, t=0 ; r=0, t>0$. When several symbolic factors of a product are identical we proceed as in the following example. Let $\lambda, \mu$ denote umbræ $(\$ 8)$, and suppose the coefficient of $x^{n}$ in $\cos ^{2} \lambda x$ is required. We write $\cos ^{2} \lambda x \equiv \cos \lambda x \cos \mu x$, find the coefficient of $x^{n}$ in $\cos \lambda x \cos \mu x$ by actual multiplication of the series for $\cos \lambda x, \cos \mu x$ or otherwise ( $\$ 24)$, and in the result, after each exponent of $\lambda, \mu$ has been degraded 
to a suffix, replace $\mu$ by $\lambda$. Once more we emphasize that in all expansions zero exponents must be included. Thus $\lambda, \mu, \cdots, \nu$ being umbræ,

$$
(\lambda+\mu+\cdots+\nu)^{n}=\sum \frac{n !}{r ! p ! \cdots t !} \lambda^{r} \mu^{p} \cdots \nu^{t}=\sum \frac{n !}{r ! p ! \cdots t !} \lambda_{r} \mu_{p} \cdots \nu_{t},
$$

the $\sum$ extending to all $r \geqq 0, p \geqq 0, \cdots, t \geqq 0$ such that

$$
r+p+\cdots+t=n \text {. }
$$

As always , $0 !=1$, and after the completion of all formal operations (multiplications, divisions, etc.) $\lambda^{r} \mu^{p} \cdots \nu^{t}$ is to be replaced by $\lambda_{r} \mu_{p} \cdots \nu_{t}$. By convention, for any particular choice of the signs,

$$
\left( \pm \lambda^{l} \pm \mu^{m} \pm \cdots \pm \nu^{n}\right)^{0}=\lambda^{0} \mu^{0} \cdots \nu^{0} \equiv \lambda_{0} \mu_{0} \cdots \nu_{0} .
$$

19. All letters $\lambda, \cdots, \pi^{\prime}$ denote umbræ, and the sets $\Lambda, \Lambda^{\prime}, Z$, $Z^{\prime} \equiv(\lambda, \mu, \cdots, \rho),\left(\lambda^{\prime}, \mu^{\prime}, \cdots, \tau^{\prime}\right),(\zeta, \xi, \cdots, \phi),\left(\zeta^{\prime}, \xi^{\prime}, \cdots, \pi^{\prime}\right)$ contain respectively $r, t, f, p$ letters. For $n \geqq 0$ write

$$
\left(\Lambda \mid \Lambda^{\prime}\right)^{n} \equiv \sum \pm\left( \pm \lambda \pm \mu \pm \cdots \pm \rho \pm \lambda^{\prime} \pm \mu^{\prime} \pm \cdots \pm \tau^{\prime}\right)^{n}
$$

in which all the exponents are symbolic, the summation extends to the $2^{r+t}$ possible combinations of signs within the parentheses, and the outer sign in each case is the product of the signs of $\lambda^{\prime}, \mu^{\prime}, \cdots, \tau^{\prime}$. The important special cases $t=0, r=0$ give respectively

$$
\begin{aligned}
& (\Lambda \mid)^{n}=\sum( \pm \lambda \pm \mu \pm \cdots \pm \rho)^{n} \\
& \left(\mid \Lambda^{\prime}\right)^{n}=\sum \pm\left( \pm \lambda^{\prime} \pm \mu^{\prime} \pm \cdots \pm \tau^{\prime}\right)^{n} .
\end{aligned}
$$

The umbral factors of $\left(\Lambda \mid \Lambda^{\prime}\right)^{n}$ are by definition $\lambda, \mu, \cdots, \rho, \lambda^{\prime}, \mu^{\prime}, \cdots, \tau^{\prime}$, and similarly for the others. If in these the letters be interpreted as ordinary quantities and the exponents as algebraic we have precisely the symmetric functions considered by Kronecker, cf. $\S 26$.

By definition the respective types of $\left(\Lambda \mid \Lambda^{\prime}\right)^{n},(\Lambda \mid)^{n},\left(\mid \Lambda^{\prime}\right)^{n}$ are $(r \mid t)$, $(r \mid 0),(0 \mid t)$, and the weight of each is $n,=$ the sum of the suffixes in the final non-symbolic forms of each. Considered as functions of $\lambda_{j}, \cdots, \tau_{k}^{\prime}$ these final forms are homogeneous of degrees $r+t, r, t$ respectively, the degree in any case being equal to the total number of letters in the bar function $\left(\Lambda \mid \Lambda^{\prime}\right)^{n}$, etc. The properties of these functions $\left(\Lambda \mid \Lambda^{\prime}\right)^{n}, \cdots$ are immediate from the expressions in $\S 20$ for their symbolic generators. From the principles of the symbolic calculus as developed by Blissard and Lucas it is evident that products, etc., of symbolic sines and cosines can be combined formally according to the rules of trigonometry, apd that the coefficients of like powers of $x$ in the several transforms thus effected of any identity are equal. By starting from the elliptic functions of a pure imaginary argument the development can be 
carried out isomorphically to the theory of the hyperbolic instead of the circular functions. This in some respects is preferable, but being committed to the other by Lucas' trigonometric generators for $B, E, G, R$ in $\S 14$ we shall not follow it.

20. Replacing each symbolic sine or cosine by its exponential equivalent we see immediately that

$2^{r+t} \cos \lambda x \cos \mu x \cdots \cos \rho x \sin \lambda^{\prime} x \sin \mu^{\prime} x \cdots \sin \tau^{\prime} x$

$$
=(-1)^{\frac{t-1}{2}} \sin \left(\Lambda \mid \Lambda^{\prime}\right) x \text { or }(-1)^{\frac{t}{2}} \cos \left(\Lambda \mid \Lambda^{\prime}\right) x
$$

according as $t$ is odd or even;

$$
2^{r} \cos \lambda x \cos \mu x \cdots \cos \rho x=\cos (\Lambda \mid) x
$$

and according as $t$ is odd or even,

$2^{t} \sin \lambda^{\prime} x \sin \mu^{\prime} x \cdots \sin \tau^{\prime} x=(-1)^{\frac{t-1}{2}} \sin \left(\mid \Lambda^{\prime}\right) x \quad$ or $\quad(-1)^{\frac{t}{2}} \cos \left(\Lambda^{\prime} \mid\right) x$, all of which are included in the first. Hence $\left(\Lambda \mid \Lambda^{\prime}\right)^{n}$ is an even function of each of the $r$ letters in $\Lambda$, and an odd function of each of the $t$ letters in $\Lambda^{\prime}$; or, $\left(\Lambda \mid \Lambda^{\prime}\right)^{n}$ of type $(r \mid t)$ is $r$-fold even and $t$-fold odd. Moreover $\left(\Lambda \mid \Lambda^{\prime}\right)^{n}$ is symmetric in the letters in $\Lambda$, also in those in $\Lambda^{\prime}$. Again, from the generators, $\left(\Lambda \mid \Lambda^{\prime}\right)^{2 n}=0$ if $t$ is odd, while $\left(\Lambda \mid \Lambda^{\prime}\right)^{2 n+1}=0$ if $t$ is even. The corresponding statements for $(\Lambda \mid)^{n},\left(\mid \Lambda^{\prime}\right)^{n}$ are included as special cases.

21. In $\left(\Lambda \mid \Lambda^{\prime}\right)^{n}$ we are concerned with (1) the umbræ $\lambda, \cdots, \tau^{\prime} ;(2)$ the type $(r \mid t)$; (3) the weight $n$. Each of these has a species of addition theorem. Denote by $(\Lambda, Z)$ the set consisting of all the letters in $\Lambda$ together with all those in $Z$, and similarly for $\left(\Lambda^{\prime}, Z^{\prime}\right)$. Then $\left((\Lambda, Z) \mid\left(\Lambda^{\prime}, Z^{\prime}\right)\right)^{n}$ is of type $((r+f) \mid(t+p))$, and is symmetric in $\Lambda, Z$, also in $\Lambda^{\prime}, Z^{\prime}$. The addition theorems with respect to types are given by the following, all of which are obvious on remarking that the generator of $\left((\Lambda, Z) \mid\left(\Lambda^{\prime}, Z^{\prime}\right)\right)^{n}$ is the product of the generators of $\left(\Lambda \mid \Lambda^{\prime}\right)^{n},\left(Z \mid Z^{\prime}\right)^{n}$, and reapplying the several cases of the generators in $\S 20$ to these products before equating coefficients of like powers of $x$. Let $(n, t, p) \equiv\left(n^{\prime}, t^{\prime}, p^{\prime}\right) \bmod 2$; then

\begin{tabular}{cll}
$\left(n^{\prime}, t^{\prime}, p^{\prime}\right)$ & $4\left((\Lambda, Z) \mid\left(\Lambda^{\prime}, Z^{\prime}\right)\right)^{n}$ & $((r+f) \mid(t+p))$ \\
\hline$(0,0,0)$ & $\left(\left(\Lambda \mid \Lambda^{\prime}\right),\left(Z \mid Z^{\prime}\right) \mid\right)^{n}$ & $((r \mid t),(f \mid p) \mid)$ \\
$(0,1,1)$ & $\left(\mid\left(\Lambda \mid \Lambda^{\prime}\right),\left(Z \mid Z^{\prime}\right)\right)^{n}$ & $(\mid(r \mid t),(f \mid p))$ \\
$(1,0, \cdot 1)$ & $\left(\left(\Lambda \mid \Lambda^{\prime}\right) \mid\left(Z \mid Z^{\prime}\right)\right)^{n}$ & $((r \mid t) \mid(f \mid p))$ \\
$(1,1,0)$ & $\left(\left(Z \mid Z^{\prime}\right) \mid\left(\Lambda \mid \Lambda^{\prime}\right)\right)^{n}$ & $\left((f \mid p) \mid\left(r^{\prime} t\right)\right)$
\end{tabular}

For example, when $n, t, p$ are all even, a function of type $((r+f) \mid(t+p))$ is expressible linearly in terms of functions of types $(r \mid t),(f \mid p)$ in the manner 
shown in the second column. The calculation of such a function is thus reduced to a series of symbolic multiplications (and subsequent additions) of functions whose types are $(r \mid t),(f \mid p)$.

22. Omitting the addition theorems for the umbræ we restrict the discussion of those for the weights to the particularly important cases of two and three letters. The general case of $n$ umbræ is treated similarly. In all that follows multiplications indicated by dots are purely symbolic. These are performed analogously to algebraic mu.tiplications but by the addition of suffixes instead of exponents. Thus

$$
\begin{aligned}
\left(\lambda_{r}+\mu_{r}\right) \cdot\left(\lambda_{t}+\mu_{t}\right)=\lambda_{r} \cdot \lambda_{t}+\lambda_{r} \cdot \mu_{t}+\mu_{r} \cdot \lambda_{t} & +\mu_{r} \cdot \mu_{t} \\
& =\lambda_{r+t}+\lambda_{r} \mu_{t}+\mu_{r} \lambda_{t}+\mu_{r+t}
\end{aligned}
$$

$\lambda_{2} \cdot \lambda_{3}=\lambda_{5} ; \lambda_{2} \cdot \lambda_{2}=\lambda_{4}$, not $\lambda_{2}^{2}$. Algebraic multiplication is a special case of this.

Taking first the case of two umbræ $\lambda, \mu$ we define auxiliary functions \{\} by

$$
\begin{gathered}
2\{\lambda, \mu \mid\}^{r} \equiv \phi^{r}+\psi^{r}, \quad 2\{\mid \lambda, \mu\}^{r} \equiv \phi^{r}-\psi^{r}, \\
\phi \equiv \lambda+\mu, \quad \psi \equiv \lambda-\mu, \quad r \geqq 0,
\end{gathered}
$$

and put $L^{r} \equiv L_{r}=\{\lambda, \mu \mid\}^{r}, M^{r} \equiv M_{r}=\{\mid \lambda, \mu\}^{r}$. Then

$$
4 L^{2 r}=(\lambda, \mu \mid)^{2 r}, \quad 4 M^{2 r}=(\mid \lambda, \mu)^{2 r}, \quad 4 M^{2 r+1}=(\lambda \mid \mu)^{2 r+1} .
$$

We have $\phi^{r}=L^{r}+M^{r}, \psi^{r}=L^{r}-M^{r}$, and hence for $t \geqq 0$,

$$
\phi^{r+t}=\left(L_{r}+M_{r}\right) \cdot\left(L_{t}+M_{t}\right), \quad \psi^{r+t}=\left(L_{r}-M_{r}\right) \cdot\left(L_{t}-M_{t}\right) .
$$

On the other hand

$$
2 L^{r+t}=\phi^{r+t}+\psi^{r+t}, \quad 2 M^{r+t}=\phi^{r+t}-\psi^{r+t} .
$$

Whence, substituting for $\phi^{r+t}, \psi^{r+t}$ and degrading exponents, we have the addition theorems

$$
L_{r+t}=L_{r} \cdot L_{t}+M_{r} \cdot M_{t}, \quad M_{r+t}=L_{r} \cdot M_{t}+M_{r} \cdot L_{t} .
$$

For example $L_{1}=\lambda_{1} \mu_{0}, L_{2}=\lambda_{2} \mu_{0}+\mu_{2} \lambda_{0}, M_{1}=\lambda_{0} \mu_{1}, M_{2}=2 \lambda_{1} \mu_{1}$; hence $L_{1} \cdot L_{2}=\lambda_{3} \mu_{0}+\lambda_{1} \mu_{2}, M_{1} \cdot M_{2}=2 \lambda_{1} \mu_{2}$. Again

$$
L_{3}=\frac{1}{2}\left[(\lambda+\mu)^{3}+(\lambda-\mu)^{3}\right]=\lambda_{3} \mu_{0}+3 \lambda_{1} \mu_{2},
$$

so that $L_{3}=L_{1} \cdot L_{2}+M_{1} \cdot M_{2}$ as required by the first of the theorems.

23. The case* of three umbræ $\lambda, \mu, \nu$ is treated in the same way, and we need

* If $\lambda, \mu, \nu$ be interpreted as ordinaries and exponents as algebraic, the special cases of the addition theorems of the first kind (in $\$ 21$ ) for the functions $\{\lambda, \mu, \nu \mid\}^{r}$, etc., which are of the same form as those for $(\lambda, \mu, \nu \mid)^{r}$, etc., have interesting consequences when $r$ is prime for Fermat's quotients. Cf. Bachmann, J o urnal für M a the matik, vol. 142 (1913), pp. 41-50; Dickson, History of the Theory of Numbers, vol. I, p. 111. 
give only the results. There can be no confusion between $\alpha, \beta, \gamma, \delta$ here and the substitutions of $\Gamma$, nor between $B$ and the Bernoulli numbers. Write

$$
\begin{array}{ll}
\alpha=\lambda+\mu+\nu, & 4 A^{r} \equiv 4\{\lambda, \mu, \nu \mid\}^{r} \equiv \alpha^{r}+\beta^{r}+\gamma^{r}+\delta^{r}, \\
\beta=\lambda-\mu+\nu, & 4 B^{r} \equiv 4\{\lambda, \mu \mid \nu\}^{r} \equiv \alpha^{r}+\beta^{r}-\gamma^{r}-\delta^{r}, \\
\gamma=\lambda+\mu-\nu, & 4 C^{r} \equiv 4\{\mid \lambda, \mu, \nu\}^{r} \equiv \alpha^{r}-\beta^{r}-\gamma^{r}+\delta^{r}, \\
\delta=\lambda-\mu-\nu, & 4 D^{r} \equiv 4\{\nu \mid \lambda, \mu\}^{r} \equiv \alpha^{r}-\beta^{r}+\gamma^{r}-\delta^{r},
\end{array}
$$

and put $A^{r}, B^{r}, C^{r}, D^{r} \equiv A_{r}, B_{r}, C_{r}, D_{r}$. We have

$$
\begin{aligned}
& 8 A^{2 r}=(\lambda, \mu, \nu \mid)^{2 r}, \quad 8 B^{2 r+1}=(\lambda, \mu \mid \nu)^{2 r+1}, \\
& 8 C^{2 r+1}=(\mid \lambda, \mu, \nu)^{2 r+1}, 8 D^{2 r}=(\nu \mid \lambda, \mu)^{2 r},
\end{aligned}
$$

and the addition theorem for $r, t \geqq 0$,

$$
\begin{aligned}
& A_{r+t}=A_{r} \cdot A_{t}+B_{r} \cdot B_{t}+C_{r} \cdot C_{t}+D_{r} \cdot D_{t}, \\
& B_{r+t}=A_{r} \cdot B_{t}+B_{r} \cdot A_{t}+C_{r} \cdot D_{t}+D_{r} \cdot C_{t}, \\
& C_{r+t}=A_{r} \cdot C_{t}+B_{r} \cdot D_{t}+C_{r} \cdot A_{t}+D_{r} \cdot B_{t}, \\
& D_{r+t}=A_{r} \cdot D_{t}+B_{r} \cdot C_{t}+C_{r} \cdot B_{t}+D_{r} \cdot A_{t} .
\end{aligned}
$$

There also are multiplication theorems for the calculation of $A_{n r}$, etc., but we shall omit these.

24. As they are frequently useful we write down the \{\} equivalents for 2 and 3 letters of the generators in $\S 20$ :

$$
\begin{aligned}
\cos \lambda x \cos \mu x & =\cos \{\lambda, \mu \mid\} x=\cos \{\mu, \lambda \mid\} x, \\
\sin \lambda x \sin \mu x & =-\cos \{\mid \lambda, \mu\} x=-\cos \{\mid \mu, \lambda\} x, \\
\cos \lambda x \sin \mu x & =\sin \{\lambda \mid \mu\} x=\sin \{\mu, \lambda \mid\} x, \\
\sin \lambda x \cos \mu x & =\sin \{\mu \mid \lambda\} x=\sin \{\lambda, \mu \mid\} x ; \\
\cos \lambda x \cos \mu x \cos \nu x & =\cos \{\lambda, \mu, \nu \mid\} x=\cos \{\{\lambda, \mu \mid\}, \nu \mid\} x, \\
\cos \lambda x \cos \mu x \sin \nu x & =\sin \{\lambda, \mu \mid \nu\} x=\sin \{\nu,\{\lambda, \mu \mid\} \mid\} x, \\
\sin \lambda x \sin \mu x \sin \nu x & =-\sin \{\mid \lambda, \mu, \nu\} x=-\sin \{\nu,\{\mid \lambda, \mu\} \mid\} x, \\
\sin \lambda x \sin \mu x \cos \nu x & =-\cos \{\nu \mid \lambda, \mu\} x=-\cos \{\{\mid \lambda, \mu\}, \nu \mid\} x .
\end{aligned}
$$

25. None of the twelve polynomials can be computed by linear recurrence, that is, by an ordinary difference equation of finite order, since otherwise an elliptic function would satisfy a linear differential equation of finite order and the first degree. We now give two symbolic linear recurrences by means of which, in conjunction with the foregoing addition theorems, the actual computation of all the polynomials can be effected systematically without reference to elliptic functions. Or, by using the results of $\$ \S 30,31$, the polynomials can 
be calculated directly from the recurrences. The addition theorems in any case shorten the labor but are not indispensable.

To find the recurrences for $L, M$ of $\S 22$ we proceed as if $\lambda, \mu$ were ordinaries, forming the equation in $w$ whose roots are $\phi, \psi$ and multiplying the result by $w^{n}$, finally degrading exponents and replacing ordinary products by dot multiplications $(\S 22)$. The formal algebraic details parallel those for the case of ordinaries, as given for example by Lucas 5, pp. 308-310, and may be omitted. For $w=$ either $L$ or $M$, and $n \geqq 0$, we find

$$
w_{n+2}-2 \lambda_{1} \cdot w_{n+1}+\left(\lambda_{2}-\mu_{2}\right) \cdot w_{n}=0,
$$

with the initial values directly from the definitions of $L, M$,

$$
L_{0}=\lambda_{0} \mu_{0}, \quad L_{1}=\lambda_{1} \mu_{0}, \quad M_{0}=0, \quad M_{1}=\lambda_{0} \mu_{1} .
$$

Thus for $n=0, w=L$, we have

$$
\begin{aligned}
L_{2}=2 \lambda_{1} \cdot L_{1}-\left(\lambda_{2}-\mu_{2}\right) \cdot L_{0} & =2 \lambda_{1} \cdot \lambda_{1} \mu_{0}-\left(\lambda_{2}-\mu_{2}\right) \cdot \lambda_{0} \mu_{0} \\
& =2 \lambda_{2} \mu_{0}-\lambda_{2} \mu_{0}+\lambda_{0} \mu_{2}=\lambda_{2} \mu_{0}+\lambda_{0} \mu_{2},
\end{aligned}
$$

which is correct. Put $n=1$ :

$$
L_{3}=2 \lambda_{1} \cdot\left(\lambda_{2} \mu_{0}+\lambda_{0} \mu_{2}\right)-\left(\lambda_{2}-\mu_{2}\right) \cdot \lambda_{1} \mu_{0}=\lambda_{3} \mu_{0}+3 \lambda_{1} \mu_{2},
$$

and so on.

Similarly if $w$ is any one of $A, B, C, D$,

$$
\begin{aligned}
& w_{n+4}-4 \lambda_{1} \cdot w_{n+3}+2\left(3 \lambda_{2}-\mu_{2}-\nu_{2}\right) \cdot w_{n+2}-4\left(\lambda_{3}-\lambda_{1} \mu_{2}-\mu_{2} \nu_{1}\right) \cdot w_{n+1} \\
& +\left(\lambda_{4}+\mu_{4}+\nu_{4}-2 \lambda_{2} \mu_{2}-2 \mu_{2} \nu_{2}-2 \nu_{2} \lambda_{2}\right) \cdot w_{\imath}=0 \text {; } \\
& A_{0}=\lambda_{0} \mu_{0} \nu_{0}, \\
& B_{0}=0 \text {, } \\
& A_{1}=\lambda_{1} \mu_{0} \nu_{0}, \\
& B_{1}=\lambda_{0} \mu_{0} \nu_{1} \text {, } \\
& A_{2}=\lambda_{2} \mu_{0} \nu_{0}+\lambda_{0} \mu_{2} \nu_{0}+\lambda_{0} \mu_{0} \nu_{2} \text {, } \\
& B_{2}=2 \lambda_{1} \mu_{0} \nu_{1} \text {, } \\
& A_{3}=\lambda_{3} \mu_{0} \nu_{0}+3 \lambda_{1} \mu_{2} \nu_{0}+3 \lambda_{1} \mu_{0} \nu_{2} \\
& B_{3}=\lambda_{0} \mu_{0} \nu_{3}+3 \lambda_{2} \mu_{0} \nu_{1}+3 \lambda_{0} \mu_{2} \nu_{1} \\
& C_{0}=0 \text {, } \\
& D_{0}=0 \text {, } \\
& C_{1}=0 \text {, } \\
& D_{1}=\lambda_{0} \mu_{1} \nu_{0} \text {, } \\
& C_{2}=2 \lambda_{0} \mu_{1} \nu_{1} \\
& D_{2}=2 \lambda_{1} \mu_{1} \nu_{0} \text {, } \\
& C_{3}=6 \lambda_{1} \mu_{1} \nu_{1} \text {; } \\
& D_{3}=\lambda_{0} \mu_{3} \nu_{0}+3 \lambda_{2} \mu_{1} \nu_{0}+3 \lambda_{0} \mu_{1} \nu_{2} \text {. }
\end{aligned}
$$

Provided multiplications be interpreted as above, viz., as dot multiplications, the theory of such symbolic recurrences is obviously identical in its formal aspects with that of ordinary recurrences. Hence for $L, M$ we have at once isomorphs of all the algebraic formulas developed by Lucas for his $U, V$. Thus, for example, $L, M$ are expressible as symbolic continuants, cf. Lucas 4 , 
p. 193. Similarly $A, B, C, D$ have symbolic properties completely analogous to the algebraic relations between any system of four independent solutions of

$$
y_{n+4}+p y_{n+3}+q y_{n+2}+r y_{n+1}+s y_{n}=0 ;
$$

in particular there are symbolic equivalents of the generalized continued fractions of Jacobi, Fürstenau, and others. This can be continued in the same way for functions of $4,5, \cdots, n$ umbræ.

There is a more general aspect of the formulas of this part which is useful elsewhere. The development of any function of $x$ which is even, odd, or arbitrary in $x$ can be written in the symbolic forms

$$
\cos \eta x, \quad \sin \zeta x, \quad \cos \eta x+i \sin \zeta x
$$

respectively, and the coefficient of $x^{n}$ in the product of any number of such functions can be most readily investigated by the symbolic trigonometry which we have sketched. When $n$ functions are concerned the symbolic recurrences are of order $n$.

One special case of interest may be mentioned. As remarked in $\S 19,(\Lambda \mid)^{n}$, $\left(\mid \Lambda^{\prime}\right)^{n}$, when exponents are interpreted as algebraic and the umbræ as ordinaries, are the symmetric functions of Kronecker 13, p. 385, which he took as his point of departure for deducing certain properties of the Bernoulli numbers. The step-by-step symbolic interpretation of Kronecker's analysis may be made without difficulty, and all his formulas translated into terms of umbræ instead of ordinaries. The formulas thus derived contain his as limiting cases. Bernoulli numbers enter Kronecker's formulas as coefficients in the expansion of hyperbolic tangents, and hence the manner in which they appear is quite distinct from that of the present discussion.

26. Corresponding to any product of elliptic functions there is a unique product of symbolic sines and cosines, and hence a unique $\left(\Lambda \mid \Lambda^{\prime}\right)^{n}$. From any elliptic identity we write down an identity between bar functions of general odd or even weight. When the identity contains ns $x$, cs $x$ or ds $x$ it is first multiplied throughout by the lowest power of $x$ such that these functions can be replaced by $x$ ns $x, x \operatorname{cs} x$ or $x$ ds $x$ respectively wherever they occur. Now the umbræ $\lambda, \mu, \cdots, \tau^{\prime}$ in $\left(\Lambda \mid \Lambda^{\prime}\right)^{n}$ are its umbral factors $(\$ 19)$, and hence the isomorphism between elliptic and polynomial identities is complete.

\section{Algebraic Relations and Congruences}

27. For $z=1$ the polynomials degenerate to Bernoulli and allied numbers, $\S 14$. Let $\omega$ denote a complex cube root of unity. The cases $z=-\omega$, $z=\frac{1}{2}$, which by an obvious analogy may be called the equianharmonic and harmonic, are noted here in passing. They are characterized by the vanishing 
of certain invariants $(\$ 4)$. Write

$$
\begin{gathered}
A_{n}(z), \alpha A_{n}(z), \beta A_{n}(z) \equiv A_{n}(z), A_{n}^{\prime}(z), A_{n}^{\prime \prime}(z), \\
I_{n}(z)=\left|\begin{array}{lll}
A_{n}(z) & A_{n}^{\prime}(z) & A_{n}^{\prime \prime}(z) \\
A_{n}^{\prime}(z) & A_{n}^{\prime \prime}(z) & A_{n}(z) \\
A_{n}^{\prime \prime}(z) & A_{n}(z) & A_{n}^{\prime}(z)
\end{array}\right| .
\end{gathered}
$$

Then from the table in $\S 2$,

$$
I_{n}(z)=\left|\begin{array}{rrr}
A_{n}(z) & A_{n}^{\prime}(z) & A_{n}^{\prime \prime}(z) \\
\alpha A_{n}(z) & \alpha A_{n}^{\prime}(z) & \alpha A_{n}^{\prime \prime}(z) \\
\beta A_{n}(z) & \beta A_{n}^{\prime}(z) & \beta A_{n}^{\prime \prime}(z)
\end{array}\right|,
$$

and from the values of $\alpha^{\prime}, \beta^{\prime}$ in $\S 1$, it is evident that this vanishes when $z=\alpha^{\prime} z, z=\beta^{\prime} z, \alpha^{\prime} z=\beta^{\prime} z$, that is, when $z=-\omega,-\omega^{2}$. Hence

$$
I_{n}(-\omega)=I_{n}\left(-\omega^{2}\right)=0,
$$

and therefore from the tables in $\$ \S 13,16$ we have, when $u=-\omega$ or $-\omega^{2}$,

$$
\begin{aligned}
& S_{2 n+1}^{3}(u)+\alpha S_{2 n+1}^{3}(u)+ \beta S_{2 n+1}^{3}(u) \\
&-3 S_{2 n+1}(u) \alpha S_{2 n+1}(u) \beta S_{2 n+1}(u)=0 \\
& P_{2 n+1}^{3}(u)+\alpha P_{2 n+1}^{3}(u)+ \beta P_{2 n+1}^{3}(u) \\
&-3 P_{2 n+1}(u) \alpha P_{2 n+1}(u) \beta P_{2 n+1}(u)=0 \\
&{ }^{C} C_{2 n}^{3}(u)+\alpha C_{2 n}^{3}(u)+\beta C_{2 n}^{3}(u)-3 C_{2 n}(u) \alpha C_{2 n}(u) \beta C_{2 n}(u)=0 \\
& \gamma C_{2 n}^{3}(u)+\epsilon C_{2 n}^{3}(u)+\delta C_{2 n}^{3}(u)-3 \gamma C_{2 n}(u) \epsilon C_{2 n}(u) \delta C_{2 n}(u)=0,
\end{aligned}
$$

the fourth of which follows from the third on transforming by $\gamma$, or it is independently obvious from the last table in $\S 16$. The product of the last two is another invariant of the same form which vanishes for the same values of $u$. This invariant corresponds to the entire last table in $\$ 16$, the determinant of which is a circulant of the sixth order, and hence, by a well known theorem of Glaisher, is expressible as a circulant of the third order. From the values of $\alpha^{\prime \prime}, \beta^{\prime \prime}$ in $\S 1$ and the form of $I_{n}(z)$ each of these invariants vanishes also when $z=0,1$. The harmonic invariants are written down in the same way.

28. Applying the first formulas of $\S 24$ to the final set in $\S 17$ we have, for $n>0$,

$$
\begin{aligned}
\{C, C \mid\}^{2 n}-\{\mid S, S\}^{2 n} & =0, \\
\{\alpha C, \alpha C \mid\}^{2}+(1-z)\{\mid \alpha S, \alpha S\}^{2 n} & =0, \\
\{\beta C, \beta C \mid\}^{2 n}+z\{\mid \beta S, \beta S\}^{2 n} & =0, \\
\{\gamma C, \gamma C \mid\}^{2 n}-z\{\mid S, S\}^{2 n} & =0, \\
\{\delta C, \delta C \mid\}^{2 n}+\{\mid \alpha S, \alpha S\}^{2 n} & =0, \\
\{\epsilon C, \epsilon C \mid\}^{2 n}-(1-z)\{\mid \beta S, \beta S\}^{2 n} & =0,
\end{aligned}
$$


in which $C=C(z)$, and likewist for the rest. For $z=1$ we get from $\S 14$ the corresponding degenerate form, $(i=\sqrt{-1})$,

$$
\{i E, i E \mid\}^{2 n}-\{\mid H, H\}^{2 n}=0, \quad \text { or } \quad(-1)^{n}\{E, E \mid\}^{2 n}=\{\mid H, H\}^{2 n},
$$

from the first or fourth of the above. The remaining degenerate forms are identities between powers of $i$. In full the degenerate relation is

$$
(-1)^{n} \sum_{r=0}^{n}\left(\begin{array}{c}
2 n \\
2 r
\end{array}\right) E_{2 n-2 r} E_{2 r}=\sum_{r=1}^{n}\left(\begin{array}{c}
2 n \\
2 r-1
\end{array}\right) H_{2 n-2 r+1} H_{2 r-1} \text {. }
$$

Similarly for the polynomials $P$ with $n>1$,

$$
\begin{gathered}
\{P, P \mid\}^{2 n}-\{\beta P, \beta P \mid\}^{2 n}=0, \quad\{P, P \mid\}^{2 n}-\{\alpha P, \alpha P \mid\}^{2 n}=0, \\
2^{2 n-2}\{B, B \mid\}^{2 n}-\{R, R \mid\}^{2 n}=0,
\end{gathered}
$$

the first of which corresponds to

$$
x^{2} \mathrm{~ns}^{2} x-x^{2} \mathrm{ds}^{2} x=x^{2} k^{2},
$$

and the second comes from this as shown in $\S 17$ by transforming with respect to $\alpha$. The degenerate relation reduces by $\S 14$ to

$$
\sum_{r=0}^{n}\left(\begin{array}{c}
2 n \\
2 r
\end{array}\right)\left(2^{2 n-2 r-1}+2^{2 r-1}-1\right) B_{2 n-2 r} B_{2 r}=0 \text {. }
$$

29. It is well known that all the algebra including the addition theorems of the elliptic functions follows from the square relations $\operatorname{cn}^{2} x+\operatorname{sn}^{2} x=1$, etc., and the expressions for the derivatives of $\operatorname{sn} x, \operatorname{cn} x$. Hence all relations between the polynomials are implicit in $\$ 28$ and the formulas next given, which are written down from the expressions for the derivatives of $\operatorname{sn} x, \operatorname{cn} x$, $x$ ns $x$ and their transforms by $\S 16$, or directly from the first member of each of the three sets as suggested at the end of $\S 17$. The first formulas of $\S 24$ are used for all, and the results may be checked at a glance by comparing with Glaisher 6, p. 92 . Thus, the dot being as in $\$ 22$, we obtain from

by differentiation,

$$
\operatorname{sn}(x, k)=\sin S(z) x,
$$

or

$$
\text { cn }(x, k) \operatorname{dn}(x, k)=S_{1}(z) \cdot \cos S(z) x,
$$

$$
\cos C(z) x \cos \gamma C(z) x=S_{1}(z) \cdot \cos S(z) x,
$$

which gives at once the first of the following:

$$
\begin{aligned}
S_{2 n+1} & =\{C, \gamma C \mid\}^{2 n}, & (2 n+1) P_{2 n+2} & =-\{\alpha P, \beta P \mid\}^{2 n+2}, \\
\alpha S_{2 n+1} & =\{\alpha C, \delta C \mid\}^{2 n}, & (2 n+1) \alpha P_{2 n+2} & =-\{P, \beta P \mid\}^{2 n+2}, \\
\beta S_{2 n+1} & =\{\beta C, \epsilon C \mid\}^{2 n} ; & (2 n+1) \beta P_{2 n+2} & =-\{P, \alpha P \mid\}^{2 n+2} ; \\
C_{2 n+2} & =\{S, \gamma C \mid\}^{2 n+1}, & \gamma C_{2 n+2} & =z\{S, C \mid\}^{2 n+1}, \\
\alpha C_{2 n+2} & =-(1-z)\{\alpha S, \delta C \mid\}^{2 n+1}, & \delta C_{2 n+2} & =-\{\alpha S, \alpha C \mid\}^{2 n+1}, \\
\beta C_{2 n+2} & =-z\{\beta S, \epsilon C \mid\}^{2 n+1} ; & \epsilon C_{2 n+2} & =(1-z)\{\beta S, \beta C \mid\}^{2 n+1},
\end{aligned}
$$


in all of which $n \geqq 0$. By means of the $L, M$ addition theorems $(\$ 22)$ and recurrences $(\$ 25)$ all of the polynomials can be calculated successively, and without excessive labor, from the initial values for $n=0,1$ (which are given by the definitions). Putting $z=1$ as before we find the degenerate forms:

$$
\begin{array}{lrl}
H_{2 n+1}=(-1)^{n}\{E, E \mid\}^{2 n}, & (2 n+1) 2^{2 n} B_{2 n+2}=-\{R, R \mid\}^{2 n+2}, \\
E_{2 n+2}=(-1)^{n+1}\{H, i E \mid\}^{2 n}, & (2 n+1) R_{2 n+2}=-\{2 B, R \mid\}^{2 n+2}
\end{array}
$$

whence, by comparison with those in $\$ 29$,

$$
H_{2 n+1}=\{\mid H, H\}^{2 n}, \quad(2 n+1) B_{2 n+2}=-\{B, B \mid\}^{2 n+2} .
$$

30. As a last example of relations involving bar functions of not more than two umbræ we take the identities which express the elliptic functions as products of two others, and those between the functions and their reciprocals. The argument is $z$, as before.

$$
\begin{aligned}
& S_{2 n+1}=\{\alpha S, C \mid\}^{2 n+1}=\{\beta S, \gamma C \mid\}^{2 n+1}, \\
& \alpha S_{2 n+1}=\{\beta S, \alpha C \mid\}^{2 n+1}=\{S, \delta C \mid\}^{2 n+1} \text { : } \\
& \beta S_{2 n+1}=\{S, \beta C \mid\}^{2 n+1}=\{\alpha S, \epsilon C \mid\}^{2 n+1} ; \\
& P_{2 n}=\{\alpha P, \delta C \mid\}^{2 n}=\{\beta P, \beta C \mid\}^{2 n}, \\
& \alpha P_{2 n}=\{\beta P, \epsilon C \mid\}^{2 n}=\{P, C \mid\}^{2 n} \text {, } \\
& \beta P_{2 n}=\{P, \gamma C \mid\}^{2 n}=\{\alpha P, \alpha C \mid\}^{2 n} ; \\
& (2 n+1) C_{2 n}=\{S, \alpha P \mid\}^{2 n+1}, \quad C_{2 n}=\{\gamma C, \epsilon C \mid\}^{2 n}, \\
& (2 n+1) \alpha C_{2 n}=\{\alpha S, \beta P \mid\}^{2 n+1}, \quad \alpha C_{2 n}=\{\delta C, \gamma C \mid\}^{2 n} \text {, } \\
& (2 n+1) \beta C_{2 n}=\{\beta S, P \mid\}^{2 n+1}, \quad \beta C_{2 n}=\{\epsilon C, \delta C \mid\}^{2 n} \text {, } \\
& (2 n+1) \gamma C_{2 n}=\{S, \beta P \mid\}^{2 n+1}, \quad \gamma C_{2 n}=\{C, \alpha C \mid\}^{2 n} \text {, } \\
& (2 n+1) \delta C_{2 n}=\{\alpha S, P \mid\}^{2 n+1}, \quad \delta C_{2 n}=\{\alpha C, \beta C \mid\}^{2 n} \text {, } \\
& (2 n+1) \epsilon C_{2 n}=\{\beta S, \alpha P \mid\}^{2 n+1}, \quad \epsilon C_{2 n}=\{\beta C, C \mid\}^{2 n} \text {; }
\end{aligned}
$$

whence the degenerate forms,

$$
\begin{aligned}
H_{2 n+1} & =(-1)^{n}\{1, E \mid\}^{2 n+1}, & \{1, E \mid\}^{2 n} & =0, \quad n>0, \\
1 & =(-1)^{n}\{H, i \mid\}^{2 n+1}, & (2 n+1) E_{2 n} & =2(-1)^{n}\{H, i R \mid\}^{2 n+1}, \\
2^{2 n-1} B_{2 n} & =\{R, 1 \mid\}^{2 n}, & \{1, R \mid\}^{2 n+1} & =0, \quad n>0, \\
2 n+1 & =\{1,2 B \mid\}^{2 n+1}, & 2 R_{2 n} & =\{2 B, E \mid\}^{2 n}
\end{aligned}
$$


31. The simplest general relations between polynomials of one kind and of several ranks are found from the differential equations for the elliptic functions. Let $\lambda, \mu, \nu$ denote umbræ and consider functions $v, t, w, u$ of $x$ such that

$$
\begin{aligned}
v & =\cos \lambda x, & v^{\prime \prime} & =a v+2 b v^{3}, \\
t & =\sin \mu x, & t^{\prime \prime} & =c t+2 d t^{3}, \\
w & =x u=\cos \nu x, & u^{\prime \prime} & =g u+2 h u^{3},
\end{aligned}
$$

in which $a, b, c, d, g, h$ are independent of $x$, and double accents denote second derivatives with respect to $x$. Then $v, t, u$ are elliptic functions, and hence $\lambda, \mu, \nu$ polynomials in $z$ ( $=$ the square of the modulus). From the differential equations for $v, t, w$ we get by substituting the symbolic trigonometric equivalents of $v, t, w$, performing the differentiations and equating coefficients of like powers of $x$,

$$
\begin{aligned}
& \lambda_{2 n+2}+a \lambda_{2 n}+2 b\{\lambda, \lambda, \lambda \mid\}^{2 n}=0, \\
& \mu_{2 n+3}+c \mu_{2 n+1}-2 d\{\mid \mu, \mu, \mu\}^{2 n+1}=0, \\
& n(2 n+1) \nu_{2 n+2}+(n+1)(2 n+1) g \nu_{2 n}-h\{\nu, \nu, \nu \mid\}^{2 n+2}=0,
\end{aligned}
$$

in which we have used the last formulas of $\$ 24$ for the coefficients* in $v^{3}, t^{3}, w^{3}$. Knowing the values of the constants in the cases $\lambda=C, \mu=S, \nu=P$ from Glaisher 6, p. 122, we write down the rest of the following table by the methods of $\S 17$. The argument is $z$ throughout.

$$
\begin{array}{lll}
\quad(\lambda, a, b)= & (\mu, c, d)= & (\nu, g, h)= \\
(C, 2 z-1,-z), & (S,-1-z, z), & (P,-1-z, 1), \\
(\alpha C,-1-z, 1), & (\alpha S, 2-z, 1-z), & (\alpha P, 2-z, 1), \\
(C, 2-z,-1+z), & \left(\beta S, 2 z-1,-z+z^{2}\right) ; & (\beta P, 2 z-1,1) . \\
(\gamma C, 2-z,-1), & & \\
(\delta C, 2 z-1,1-z), & \\
(\epsilon C,-1-z, z) ; &
\end{array}
$$

We omit the degenerate forms. The bar functions of $\lambda, \mu, \nu$ may be computed by the addition theorems of $\S 23$ and the recurrences of $\S 25$, and hence each of the polynomials for all ranks can be calculated independently of the remaining polynomials. In using $\$ \S 23,25$ we perform all operations for three distinct umbræ, putting these equal to one another in the successive final steps (see $\S 18$ ). We must pass on to a brief discussion of the congruences for prime moduli.

\footnotetext{
* The differential equation for $w$ is $x^{2} w^{\prime \prime}=2 x w^{\prime}+\left(g x^{2}-2\right) w+h u^{3}$.
} 
32. Henceforth $p$ denotes an odd prime $>0$. (Some of the congruences are also valid modulo 2 , but this case is of such slight interest that we ignore it.) The theorem of arithmetic which gives the congruences modulo $p$ is due to Lucas 4, pp. 229-230:

$$
\begin{aligned}
&\left(\begin{array}{c}
m \\
n
\end{array}\right) \equiv\left(\begin{array}{c}
m_{1} \\
n_{1}
\end{array}\right)\left(\begin{array}{c}
m_{1}^{\prime} \\
n_{1}^{\prime}
\end{array}\right) \\
& m=m_{1} p+m_{1}^{\prime}, \quad n=n_{1} p+n_{1}^{\prime}, \quad 0 \leqq m_{1}^{\prime}, \quad n_{1}^{\prime}<p .
\end{aligned}
$$

We shall in future omit the " $\bmod p$ " in writing congruences, and use " $\equiv "$ only in the sense of congruence modulo $p$. From Lucas' theorem it is easy to infer that $, \lambda, \mu$ denoting umbræ of integers and the significance of the dot being as in $\$ 22$,

$$
(\lambda \pm \mu)^{r p+s} \equiv\left(\lambda^{p} \pm \mu^{p}\right)^{r} \cdot(\lambda \pm \mu)^{s}, \quad 0 \leqq s<p,
$$

the upper signs or the lower being taken throughout. By repeated application of this we find the following. Let

$$
N=r_{n} p^{n}+r_{n-1} p^{n-1}+r_{n-2} p^{n-2}+\cdots+r_{1} p_{1}+r_{0}
$$

be the (unique) expression of $N$ in the scale of $p$, so that $0 \leqq r_{j}<p(j=0,1$, $\cdots, n)$. Then

$$
\begin{aligned}
& (\lambda+\mu)^{N} \equiv\left(\lambda^{p^{n}}+\mu^{p^{n}}\right)^{r_{n}} \cdot\left(\lambda^{p^{n-1}}+\mu^{p^{n-1}}\right)^{r_{n-1}} \cdots\left(\lambda^{p}+\mu^{p}\right)^{r_{1}} \cdot(\lambda+\mu)^{r_{0}}, \\
& (\lambda-\mu)^{N} \equiv\left(\lambda^{p^{n}}-\mu^{p^{n}}\right)^{r_{n}} \cdot\left(\lambda^{p^{n-1}}-\mu^{p^{n-1}}\right)^{r_{n-1}} \cdots\left(\lambda^{p}-\mu^{p}\right)^{r_{1}} \cdot(\lambda-\mu)^{r_{0}} .
\end{aligned}
$$

Denote the right-hand members of these congruences by

$$
\prod_{j=0}^{n}\left(\lambda^{p^{n-j}} \pm \mu^{p^{n-j}}\right)^{\tau_{n-j}}
$$

respectively, in which the accented $\Pi^{\prime}$ indicates that the products of the several factors are to be performed as dot multiplications. Then

$$
\begin{aligned}
& \{\lambda, \mu \mid\}^{N} \equiv \frac{1}{2} \prod_{j=0}^{n}\left(\lambda^{p^{n-j}}+\mu^{p^{n-j}}\right)^{r_{n}-j}+\frac{1}{2} \prod_{j=0}^{n}\left(\lambda^{p^{n-j}}-\mu^{p^{n-j}}\right)^{r_{n-j}}, \\
& \{\mid \lambda, \mu\}^{N} \equiv \frac{1}{2} \prod_{j=0}^{n}\left(\lambda^{p^{n-j}}+\mu^{p^{n-j}}\right)^{r_{n-j}}-\frac{1}{2} \prod_{j=0}^{n}\left(\lambda^{p^{n-j}}-\mu^{p^{n-j}}\right)^{r_{n-j}}
\end{aligned}
$$

The distributed non-symbolic equivalent of each right-hand me mber after the performance of all dot multiplications and the degradation of all exponents is a quadratic form with positive integral coefficients of the form

$$
\left(\begin{array}{l}
r_{n} \\
r_{n}^{\prime}
\end{array}\right)\left(\begin{array}{l}
r_{n-1} \\
r_{n-1}^{\prime}
\end{array}\right) \cdots\left(\begin{array}{l}
r_{0} \\
r_{0}^{\prime}
\end{array}\right) \quad\left(r_{n}^{\prime} \leqq r_{n}, \quad r_{n-1}^{\prime} \leqq r_{n-1}, \cdots, \quad \cdots \leqq r_{0}^{\prime}\right),
$$


and the term of which this is the coefficient is

$$
\lambda_{N-N^{\prime}} \mu_{N^{\prime}} \quad\left(N^{\prime}=r_{n}^{\prime} p^{n}+r_{n-1}^{\prime} p^{n-1}+\cdots+r_{0}^{\prime}\right) .
$$

That the coefficients are positive is evident from the left-hand members in which all coefficients are such; the coefficients on the right are the positive residues modulo $p$ of those on the left.

If $\rho_{N}=\{\lambda, \mu \mid\}^{N}$, or if $\rho_{N}=\{\mid \lambda, \mu\}^{N}$, the above congruences are applicable in an obvious manner, equalities of this sort having been considered in the preceding sections. From $\S 15$ it is clear that any congruence between polynomials (the $P$ set does not immediately enter the discussion since the coefficients are not integers) is equivalent to a system of congruences between the linear functions of the form in $\S 3$ into which $H, E$ and their anharmonic transforms (the degenerate forms of the corresponding polynomials) for different ranks are partitioned.

Two important special cases of the general formula in this section are

$$
\{\lambda, \mu \mid\}^{p} \equiv \lambda_{p} \mu_{0}, \quad\{\mid \lambda, \mu\}^{p} \equiv \lambda_{0} \mu_{p}
$$

Hence in all cases we have the residue of a bar function of two integral umbræ when the rank of the function is equal to or greater than the modulus. The case of two umbræ will be completed for a prime modulus when we find, as next, the residue when the rank is less than the modulus.

33. Restating another theorem of Lucas 4, p. 229, in a form adapted to our purpose we have

$$
\begin{array}{r}
\left(\begin{array}{c}
p-1 \\
n
\end{array}\right) \equiv(-1)^{n}, \quad\left(\begin{array}{c}
p-r \\
n
\end{array}\right) \equiv(-1)^{n}\left(\begin{array}{c}
n+r-1 \\
r-1
\end{array}\right) \\
(0<r<p, \quad n<p-r) .
\end{array}
$$

Hence for $r>0$,

$$
\begin{aligned}
& (\lambda+\mu)^{p-r} \equiv \sum_{s=0}^{p-r}(-1)^{s}\left(\begin{array}{c}
r+s-1 \\
s
\end{array}\right) \lambda_{p-r-s} \mu_{s}, \\
& (\lambda-\mu)^{p-r} \equiv \sum_{s=0}^{p-r}\left(\begin{array}{c}
r+s-1 \\
s
\end{array}\right) \lambda_{p-r-s} \mu_{s}
\end{aligned}
$$

and hence, including all cases (cf. $\S 24$ ), for $h \geqq 0$,

$$
\begin{aligned}
& \{\lambda, \mu \mid\}^{p-2 h-1} \equiv \sum_{s=0}^{(p-2 h-1) / 2}\left(\begin{array}{c}
2 h+2 s \\
2 s
\end{array}\right) \lambda_{p-2 h-2 s-1} \mu_{2 s}, \\
& \{\lambda, \mu \mid\}^{p-2 h-2} \equiv \sum_{s=0}^{(p-2 h-3) / 2}\left(\begin{array}{c}
2 h+2 s+1 \\
2 s
\end{array}\right) \lambda_{p-2 h-2 s-2} \mu_{2 s},
\end{aligned}
$$




$$
\{\mid \lambda, \mu\}^{p-2 h-1} \equiv-\sum_{s=0}^{(p-2 h-1) / 2}\left(\begin{array}{c}
2 h+2 s-1 \\
2 s-1
\end{array}\right) \lambda_{p-2 h-2 s} \mu_{2 s-1} .
$$

34. The congruences in $\S 33$ admit of immediate generalization to any number $m$ of umbræ $\lambda, \rho, \cdots, \mu$. It is easily seen in the same way, or as a consequence of the fundamental formula of $\S 33$,

that we have

$$
(\lambda+\mu)^{r p+s} \equiv\left(\lambda^{p}+\mu^{p}\right)^{r} \cdot(\lambda+\mu)^{s},
$$

$$
(\lambda+\rho+\cdots+\mu)^{r p+s} \equiv\left(\lambda^{p}+\rho^{p}+\cdots+\mu^{p}\right)^{r} \cdot(\lambda+\rho+\cdots+\mu)^{s} ;
$$

and hence in the previous notation,

$$
(\lambda+\rho+\cdots+\mu)^{N} \equiv \prod_{j=0}^{n}\left(\lambda^{p^{n-j}}+\rho^{p^{n-j}}+\cdots+\mu^{p^{n-j}}\right)^{\tau_{n-j}},
$$

with $2^{m}-1$ similar congruences in which the signs of some or all of $\lambda, \rho, \cdots, \mu$ are changed throughout. From these the congruences for the general bar functions of $\S 19$ follow at once. The particular case $m=3$ is of interest in connection with the formulas of $\S 32$, but the length of this paper precludes further discussion. From the general formulas given there is no difficulty in writing down the congruences for bar functions of two or three letters from the algebraic relations developed earlier in the paper. For $z=1$ some of the degenerate cases are well known, and hence provide checks. The other degenerate cases may be checked directly in a similar manner.

\section{REFERENCES}

1. J. Blissard: Theory of generic equations, Quarterly Journal of Mathem a t i c s , vol. 4 (1861), pp. 279-305; vol. 5 (1862), pp. 58-75, 185-208.

2. J. BuISSARD: Examples of the use and application of the representative notation, ibid., vol. 6 (1864), pp. 49-64.

3. J. Blissarn: Researches in analysis, ibid., vol. 7 (1865), pp. 155-170, 223-226.

4. E. Lucas: Théorie des fonctions numériques simplement périodiques, A merican Journal of Mathematic s, vol. 1 (1878), pp. 184-238, 289-321.

5. E. LuCAs: Théorie des Nombres, 1891.

6. J. W. L. Glaisher: On elliptic functions, $\mathrm{M}$ e s s e nge $\mathrm{r}$ of $\mathrm{M}$ a t h e mati cs, vol. 11 (1881-1882), pp. 81-95, 120-138.

7. J. W. L. GLaisher: On the Bernoullian function, Q u a r terl y J o u r nal of $\mathrm{M}$ a the m a t i c s , vol. 29 (1898), pp. 1-168 (especially pp. 122-168).

8. J. W. L. GLarsher: On a class of relations connecting any $n$ consecutire Bernoullian functions, ibid., vol. 42 (1911), pp. 86-157.

9. A. CAYLEy: An Elementary Treatise on Elliptic Functions, Edition 2 (1895).

10. C. Hermite: Remarques sur le développement de cos am $x, \mathrm{P}$ a $\mathrm{r}$ i $\mathrm{C}$ o $\mathrm{m}$ p t e s $\mathrm{R}$ e $\mathrm{n} d \mathrm{u}$ s, vol. 67 (1863), p. 613; Journal de Mathématiques pures et app l i q u é e s , (2) vol. 9 (1864), p. 289; CEuvres, vol. 2, pp. 265-270.

11. C. Hermite: Sur les développements de $F(x)=\mathrm{sn}^{a} x \mathrm{cn}^{b} x \mathrm{dn}^{c} x$ où les exposants sont entiers, Académie royale des Sciences de Stockholm, Bihang III, No. 10 (1875), pp. 3-10; CEuvres, vol. 3, pp. 222-235. 
12. C. Hermite: Sur le développement des fonclions elliptiques, J o u r n a l f ü r d i e r e i n e und angewandte $M$ at hematik, vol. 81 (1876), pp. 220-228; Euvres, vol. 3, pp. 236-245.

13. L. KRONECKER: Sur quelques fonctions symétriques et sur les nombres de Bernoulli, Journal de Mathématiques puresetappliquées, (2) vol. 1 (1856), pp. 385-391.

14. L. Kronecker: Ueber die Bernoullischen Zahlen, J o u r a l f ür die reine und angew and te $M$ a the matik, vol. 94 (1883), pp. 268-269.

UNIVERSITY OF WASHINGTON,

Seattue, Wash. 